\title{
Artículos
}

\section{Diseño metodológico para estimar indicadores de accesibilidad en entornos periféricos de una zona metropolitana}

\section{Methodological procedure to compute the accessibility in the peripheral areas of a metropolitan zone}

\author{
Saúl Antonio Obregón Biosca* \\ Marco Antonio Ángeles Espinosa**
}

\section{Resumen}

La accesibilidad es una parte integral en la planificación del transporte, el cual impacta en nuestra calidad de vida. El caso de estudio de este trabajo es la accesibilidad de las periferias en los ámbitos metropolitanos. Debido a la diversidad de resultados, dependiendo del indicador de accesibilidad y de los insumos empleados, la investigación propone un procedimiento metodológico que considera los indicadores de separación e interacción espacial para analizar la accesibilidad por medio de transporte (automóvil y autobús), contrastando los resultados con las tasas promedio de viajes. Considerando el procedimiento, los resultados muestran que el indicador de interacción espacial refleja resultados más acordes en la periferia urbana.
\end{abstract}

Palabras clave: accesibilidad; viajes; periferias urbanas; automóvil; autobús.

\begin{abstract}
Accessibility is a key factor in transport planning, and the transport system impacts the life quality, being the study case the urban peripheries accessibility in a metropolitan zone. Due the results diversity, according to the accessibility indicators and inputs used, the research proposes a methodological procedure considering the

* Universidad Autónoma de Querétaro, Facultad de Ingeniería. Dirección postal: Cerro de las Campanas s/n, Las Campanas, 76010, Santiago de Querétaro, Querétaro, México. Correo electrónico: saul.obregon@uaq.mx

** Universidad Autónoma de Querétaro, Facultad de Ingeniería, Laboratorio de Estudios Viales y Movilidad. Correo electrónico: marco.angeles89@gmail.com

Nota de los autores: Agradecemos las valiosas sugerencias y los comentarios de los dictaminadores anónimos en la versión previa del documento.
\end{abstract}

D.R. (C) 2018. Estudios Demográficos y Urbanos Licencia Creative Commons Atribución-NoComercial-SinDerivar (CC BY-NC-ND) 4.0 Internacional 
separation and spatial interaction indicators, in order to analyze the accessibility by transport means (car and public bus), contrasting the results with trip generation rates. Considering the procedure and contrast, the results show that the spatial interaction approach describes better the accessibility by transport mean in the urban periphery.

Keywords: accessibility; travels; urban sprawl; automobile; bus.

\section{Introducción}

Los sistemas de transporte impactan en nuestra calidad de vida, mientras que la accesibilidad es una parte integral de la planificación y la evaluación del transporte (Obregón et al., 2016a). La accesibilidad se ha analizado desde hace tiempo a partir de varias perspectivas, tanto en lo referente a su definición como en las herramientas para su medición, enfocándose principalmente en lo que se refiere a la oportunidad que posee una persona, en un punto determinado, para participar en cierta actividad o conjunto de actividades (Thakuriah, 2001). La diversidad en los indicadores de accesibilidad y en sus insumos induce a que se arrojen resultados disímiles, por lo que dependiendo de la situación y su propósito es necesario definir el indicador que se empleará (Guy, 1983; Handy y Neimeier, 1997). En este sentido, el presente trabajo muestra un proceso metodológico que abarca la estimación de los insumos para la obtención de dos indicadores de accesibilidad (interacción espacial y separación espacial), aplicados a los entornos periféricos de un área metropolitana y comparando sus resultados, con el objetivo de analizar la accesibilidad por modo de transporte (automóvil y autobús público) en el área periférica de la Zona Metropolitana de Querétaro, partiendo del supuesto de que la accesibilidad en tal área define la elección en el modo de transporte público (autobús). Los resultados de cada indicador se comparan con la tasa media de generación de viajes por medio de transporte de cada zona periférica, así como con el ingreso familiar promedio; con ello, se analizan los resultados de cada indicador por medio de transporte. Lo anterior tiene motivación en la problemática expuesta por Guy (1983) y Handy y Neimeier (1997). Entre las fortalezas del procedimiento metodológico destaca la consideración de encuestas de origen-destino para estimar la interacción entre zonas a partir de la estimación de un modelo gravitacional y el costo generalizado del viaje por medio de transporte como medida de separación. Considerando lo anterior, se determina el indicador que mayor fortaleza refleja, en el sentido de que una mayor accesibilidad presenta relación directa con la tasa promedio de generación de viajes y con el ingreso promedio 
(reportado para el caso de estudio en Obregón y Betanzo, 2015; Obregón et al., 2015; Obregón et al., 2016b). A partir de dichas estimaciones se define el indicador de accesibilidad aplicado a entornos periféricos que refleja mayor relación directa con los supuestos anteriores. Se propone que, para estimar la accesibilidad en las periferias urbanas de una zona metropolitana, se empleen secciones censales y se realice el análisis considerando los principales medios de transporte, la infraestructura vial y los sistemas de transporte, así como la información de origen-destino, mediante el indicador de interacción espacial.

Para contextualizar el marco territorial, Bruekner y Fansler (1983) definen que el proceso de expansión gradual de la urbanización ha dado paso al término "dispersión urbana"; en dicho contexto, el proceso de periurbanización se caracteriza por asentamientos más allá de las áreas urbanas (Zasada et al., 2011; Haller, 2014), caracterizado por pasar por espacios de suelo no urbanizado de una zona a otra (Hamidi y Ewing, 2014; Zhang, 2004; Bhatta, 2010). La región periurbana es a menudo una zona de urbanización caótica que conduce a la expansión; Friedmann (2011) sostiene que tal región es una "zona de encuentro, conflicto y transformación que rodea a las grandes ciudades". Respecto a la accesibilidad, el CEAPAT (1996: 7) menciona que "les permite a las personas participar en las actividades sociales y económicas para las que se ha concebido el entorno construido". La participación ciudadana en las periferias urbanas depende de la accesibilidad, tal como sostienen Blanco et al. (2012), pues ésta será menor cuanto menos accesibilidad o integración funcional presenten, debido a que una mayor dedicación en el tiempo de los desplazamientos va en decremento de la participación. Por lo anterior, su ausencia posibilitará la discriminación, en el sentido de marginación y disminución del bienestar o calidad de vida de las personas (Domínguez et al., 2011).

Se han reportado investigaciones en donde se analiza la accesibilidad considerando la dinámica espacio-temporal, como la de Miller (1999), quien relaciona las interacciones entre el rendimiento del sistema de transporte y la localización de las actividades del individuo. Berechman y Paaswell (2001) exponen que la tasa de actividad se ve afectada por la accesibilidad a los lugares de empleo; por ello, acciones encaminadas a mejorar la accesibilidad en un área dada (por ejemplo, inversión en infraestructura), darán como resultado una mayor participación laboral, sin descuidar las características socioeconómicas y de ubicación. 


\section{Forma urbana, transporte y accesibilidad}

Dada la distribución de las áreas urbanas se induce la separación de puntos de origen y destino, mientras los desplazamientos son resultado de la ubicación del hábitat y de las actividades (Valero, 1984). En este sentido, Gutiérrez y García (2006) exponen que las transformaciones territoriales en las que están inmersos los espacios metropolitanos producen un número de destinos más dispersos y alejados del hogar. A partir de lo anterior, se establece la conexión entre la estructura espacial urbana y el transporte, la cual se indica en la duración y la distancia del viaje. Paaswell y Zupan (2007) afirman que en dichos espacios es necesaria una alta accesibilidad, que debería ser proporcionada por los sistemas de transporte. Respecto a la estructura espacial, Fuentes (2009) sostiene que en ella intervienen factores como el valor del suelo, la densidad de la población, la tenencia de la vivienda, el balance entre empleos y viviendas y la proporción de viviendas alquiladas.

Miller y Wu (2000) mencionan que el comportamiento en los desplazamientos se centra en la comprensión de procesos y decisiones de un individuo, a partir del análisis de los factores que determinan la actividad elemental de cada viaje. En consecuencia, dentro de la planificación del transporte se consideran los métodos de forma; es decir, cómo o cuándo las actividades de viaje tendrán lugar a lo largo del sistema de transporte, enfatizando la accesibilidad con enfoques alternativos para el comportamiento de los viajes en el rendimiento de dicho sistema. En este sentido, la disposición de la accesibilidad para evaluar la libertad de un individuo para participar en ciertas actividades en un ambiente de viaje determinado, es posible centrarla en las oportunidades y el costo del viaje.

$\mathrm{Al}$ analizar los sistemas de transporte urbano y regional es fundamental el análisis de su accesibilidad, debido a la creciente complejidad de los sistemas de transporte y su impacto en la calidad de vida de los individuos. Por lo anterior, es común el empleo de parámetros de accesibilidad en la planificación y la evaluación del transporte; en este sentido Alonso (2007) sostiene que la accesibilidad comprende la planeación eficaz de una ciudad, la cual requiere información útil y precisa para generar situaciones de tendencia futura. Sin embargo, existen diversas perspectivas sobre la definición y medición de la accesibilidad.

En dicho sentido, desde hace varias décadas el concepto de accesibilidad ha generado atención en diversas disciplinas, y una definición única de este concepto no parece existir, pues varía acorde con la disciplina. Para el enfoque de la presente investigación, su definición debe ir ligada con las zonas de la ciudad en estudio y las unidades de servicio, permitiéndonos medirlas. 
Por ello, de acuerdo a la definición de Goodall (1987) "la accesibilidad es la facilidad con la que se puede alcanzar un cierto sitio (destino), desde otros puntos en el territorio (orígenes), por lo que sintetiza las oportunidades de contacto e interacción entre determinados orígenes y destinos". En el mismo sentido, Johnston (2009) considera la accesibilidad como "la oportunidad de interacción y contacto entre orígenes y destinos", de acuerdo con lo expuesto por Thakuriah (2001), quien centra el concepto de accesibilidad en la oportunidad de que un individuo en un lugar determinado tenga participación en cierta actividad o conjunto de ellas. A partir de dichas definiciones resaltan dos aspectos: el primero, la probabilidad de alcanzar algún servicio entre orígenes y destinos; el segundo, la interacción entre individuos y destinos.

\subsection{El sistema de transporte y la accesibilidad}

Respecto a la importancia de la disponibilidad y posibilidad de cada ciudadano para movilizarse y acceder a sus necesidades en una ciudad o región, Pardo (2005) establece que el sistema de transporte debe resolver las necesidades principales de los ciudadanos, como acceder a la alimentación, la salud, el trabajo, entre otras. En el mismo sentido, Ozbay et al. (2006) exponen que un sistema de transporte fiable e infraestructura eficiente son necesarios para el bienestar económico de la población, ya que proporcionan un acceso adecuado a la región y permiten el funcionamiento del comercio, la industria, el trabajo y la vivienda. Respecto a las personas con discapacidad, Alonso (2007) menciona que la falta de accesibilidad a los distintos entornos, productos y servicios discrimina a los individuos con discapacidad o de edad avanzada, y expone argumentos para mejorar la accesibilidad con la finalidad de lograr una mejor interacción entre dichas personas y el entorno, mediante infraestructuras y sistemas de transporte accesibles.

\subsection{Los entornos rurales y su accesibilidad}

Los entornos rurales reflejan problemas relacionados con la accesibilidad, el transporte y la movilidad a los centros de servicio. Nutley (2003) remarca la importancia que tiene la ausencia del transporte público en entornos rurales, lo que induce a la participación de vehículos privados. Lo anterior influye en la generación de disparidades interurbanas en los tiempos de viaje, principalmente por motivos de trabajo, entre los vehículos privados y el transporte público. Al respecto Fuentes (2009) sostiene que dichas dispari-

Estudios Demográficos y Urbanos, vol. 33, núm. 1 (97), 2018, pp. 111-147 ISSN 0186-7210; e-ISSN 2448-6515; doi: http://dx.doi.org/10.24201/edu.v33i1.1740 
dades son producto de la accesibilidad diferenciada a los centros laborales, ya que mediante un análisis espacial se determina que la accesibilidad, el valor del suelo y los puestos de trabajo en el sector secundario son las variables que explican los incrementos en los tiempos de traslado. En su investigación reflexiona respecto a la localización de las actividades, pues en los núcleos centrales se erogan rentas superiores frente a las localizadas en la periferia; sin embargo, el trabajador paga un alto costo por el transporte, lo cual es comprobado por Obregón et al. (2016b), quienes estiman los costos generalizados por viaje, las emisiones y el consumo de carburante generados por la dispersión urbana. En el mismo sentido, Currie et al. (2009) reportan que la tenencia de automóviles es mucho mayor en los hogares de ámbitos periféricos, concluyendo que en ello influye la exclusión social y la dependencia del automóvil.

\subsection{Casos de estudio}

Kwan (1998) compara doce indicadores de interacción espacial, seis de oportunidades acumulativas y doce espacio-temporales, observando correlaciones altas entre los de interacción espacial y los de oportunidades acumulativas, y bajas correlaciones entre los anteriores frente a los espaciotemporales. Otra investigación en la que se evalúan los indicadores de accesibilidad es la realizada por Baradaran y Ramjerdi (2001), quienes concluyen que existen dos cuestiones fundamentales, el para quién y el para qué, por lo que una descripción clara es la condición de conectividad. A partir de lo anterior, suponen que un lugar es accesible si está conectado a otro mediante una red y medios de transporte; por lo tanto, el indicador de accesibilidad puede describir las características de la infraestructura física y con ello la cobertura de un modo específico. Sostienen que ambas cualidades (accesibilidad y conectividad) no requieren una forma binaria (que la ubicación esté conectada o no), pues pueden generarse a partir de los diferentes enlaces y los modos con los que cuenta la ubicación.

En términos de la planificación, el análisis de la accesibilidad de Garrocho y Campos (2006) -cuyo caso de estudio es el Área Metropolitana de Toluca, México- desarrolla un indicador de accesibilidad que considera parámetros de disponibilidad, costos de transporte, sensibilidad al costo y relación distancia-costo, los cuales comprobaron su utilidad como herramientas de análisis y planeación para ciudades mexicanas, ya que permiten simular escenarios con la finalidad de apoyar la toma de decisiones y definir políticas públicas. 


\section{Enfoques en los indicadores de la accesibilidad}

Existe variedad en los indicadores de la accesibilidad, por lo que se deben considerar el objetivo y los parámetros que se incorporan en cada uno de ellos con la finalidad de elegir el más consistente y preciso para el objeto de estudio. Guy (1983) advierte que los resultados pueden diferir notablemente según el indicador que se utilice; por su parte, Handy y Neimeier (1997) sostienen que lo más recomendable es que la situación y el propósito del análisis definan el tipo de indicador que se seleccione.

Respecto a la variedad de los indicadores de accesibilidad, Garrocho y Campos (2006) estipulan que éstos pueden clasificarse en cinco categorías: i) de separación espacial, los cuales se centran en promediar la distancia de los desplazamientos entre orígenes y destinos y la sensibilidad de la demanda ante cambios en el costo del transporte (fricción de la distancia); ii) de interacción espacial, que estiman la atracción de las unidades de servicio considerando como parámetros espaciales el tiempo, la distancia y el costo, entre otros, representados a partir de una función de fricción entre zonas (la atracción entre zonas es considerada como un factor); iii) de oportunidades acumulativas, que consideran la localización de los destinos como unidades de servicio, y el tiempo de desplazamiento o acceso a los destinos potenciales como medida de accesibilidad; iv) de utilidad, a partir de la percepción individual de cada usuario a cada unidad de servicio como destino; y $v$ ) de espacio-temporal, basados en las restricciones temporales de los individuos (como usuarios potenciales) ya que éstos sólo tienen periodos limitados para realizar ciertas actividades, es decir, a mayor tiempo de viaje menor tiempo para realizar actividades, por lo que consideran las limitaciones en el desempeño del individuo (tiempo para descansar, dormir o comer, así como las diferencias entre edades). Se contemplan a la vez restricciones denominadas "de autoridad", como los mandatos superiores, legales o normativos que inhiben el movimiento o actividad. Dichos autores reportan las expresiones funcionales básicas de cada categoría.

Las cinco categorías de accesibilidad se refieren en común a la población, a las unidades de servicio o a ambas, tal como expone Bach (1981), mientras Garrocho y Campos (2006) definen como componentes el físico, que es el parámetro de la distancia geográfica entre la oferta y la demanda (unidades de servicio-usuarios), y el social, que es el parámetro de distancia social entre el servicio y el usuario (la accesibilidad revelada a los servicios); en este último se recomienda el empleo de encuestas (Joseph y Phillips, 1984). Sin embargo, Geurs y Ritsema van Eck (2001) definen cuatro componentes más específicos: $i$ ) el de transporte, que es el beneficio de un individuo al

Estudios Demográficos y Urbanos, vol. 33, núm. 1 (97), 2018, pp. 111-147 ISSN 0186-7210; e-ISSN 2448-6515; doi: http://dx.doi.org/10.24201/edu.v33i1.1740 
cubrir una distancia entre un origen y un destino, considera el tiempo de viaje, los costos y el nivel de confort; ii) de uso de suelo, que explica la distribución geográfica de las actividades; iii) temporal, que muestra las oportunidades durante el día y el tiempo disponible para las actividades; y iv) individual, que expone las necesidades a partir de las características socioeconómicas de los individuos, sus oportunidades y sus habilidades, para reflejar cómo dichos parámetros influyen en el nivel de acceso al transporte y las habilidades para calificar en puestos de trabajo cercanos a la residencia.

Además de los componentes y categorías expuestas, existen otros enfoques. Arentze et al. (1994) y Handy y Niemeier (1997) reportan tres: i) el del costo del viaje, que mide la facilidad para llegar mediante un sistema de transporte (a partir del costo del viaje) a las actividades en los diversos usos de suelo; ii) el de gravedad, basado en el comportamiento de los desplazamientos, hace una analogía al modelo físico de gravedad donde la interacción de las masas esté disponible para los consumidores, sean elegidos o no; y iii) el basado en las restricciones, el cual sostiene que el individuo presenta dimensión espacial y temporal, pues las limitaciones en los viajes no se ven afectadas únicamente por la distancia, sino también por el tiempo disponible de la persona.

En este último sentido, Miller y Wu (2000) sostienen que el espaciotiempo delimita los destinos que un individuo puede alcanzar, considerando la duración de las actividades obligadas y los tiempos de viaje que dependen del sistema de transporte; se basan en el enfoque de Lenntorp (1976), quien muestra la evaluación de conjuntos de trayectorias para calcular destinos, considerando los horarios de actividad y las restricciones espaciales.

A partir de dicha reflexión, Miller y Wu (2000) proponen dos enfoques espacio-temporales en los indicadores de accesibilidad: $i$ ) el de utilidad excedente, que incorpora las características individuales de cada viajero, quien elige la que maximiza su utilidad (considera la teoría de utilidad aleatoria propuesta por McFadden, 1974); y ii) el compuesto, que combina el componente espacio-temporal y los modelos de utilidad, es decir, la utilidad de llevar a cabo viajes discrecionales considerando la movilidad obligada y el tiempo (disponible y de viaje).

Ahora bien, los indicadores de accesibilidad deben cumplir con criterios que determinen su consistencia. En este sentido, Weibull (1976) y Morris et al. (1979) establecen que: $i$ ) el orden de la información de origen-destino no afecta al indicador; ii) la respuesta del indicador no debe incrementarse a partir de los costos del transporte ni reducirse al incrementar la magnitud de la oferta; iii) es requisito que considere los patrones espaciales de los individuos; iv) debe ser viable operativamente (no ser un ejercicio académico); 
y v) de fácil interpretación de los resultados. Bath et al. (2000) sostienen que los criterios expuestos son referentes fundamentales para el diseño de indicadores de accesibilidad.

A partir de lo expuesto, diferentes factores son requeridos dependiendo del indicador, como la disponibilidad de información, tecnología y capacidades técnicas, mientras el objetivo de análisis define la selección del más adecuado para cada situación. Por lo anterior, Handy y Clifton (2001) recomiendan considerar: i) los factores de importancia para la población, ii) la disponibilidad de la información, iii) la capacidad técnica de los planificadores para dar sentido a la información, y $i v$ ) la zonificación, que permite el análisis detallado de las características cualitativas del entorno.

\subsection{Las medidas de separación espacial}

Harris (2001) propone que para medir la accesibilidad zona por zona, el análisis debe centrarse en las regiones metropolitanas, dividiendo zonas ya sea por tráfico, secciones censales, o agrupamientos de ambas. Sobre la información necesaria para cada zona, se puede incluir al menos una de las siguientes variables: establecimientos, puestos de trabajo, casas, viviendas, instalaciones que sirven de compras o de recreo, así como servicios de salud y seguridad pública. Respecto al grado de separación espacial entre lugares, Bruinsma y Rietveld (1998) mencionan que deben ser empleados parámetros comunes, como la distancia de viaje, el tiempo de traslado o el costo generalizado del viaje. Respecto a la atracción entre zonas, ésta debe representar la utilidad potencial de las oportunidades hacia un destino, en términos del beneficio que produzca en un individuo el visitar un lugar específico o un conjunto; así definen a la población como la variable de atracción. En cuanto a la elección de la zona y su escala, Arbia (1989) sustenta que la escala debe estar relacionada con el número de unidades en el área de estudio, ya que algunas ubicaciones pueden ser agregadas si presentan medidas de accesibilidad similares; la zona de estudio definirá la elección o exclusión de unidades, en este sentido, la exclusión de alguna puede afectar los resultados.

Miller (1999) reporta la aplicación del método de prisma espacio-temporal (que integra la extensión espacial de los viajes y el tiempo disponible de la participación en base a los horarios de actividades individuales), mientras Hägerstrand (1975) discute que la principal debilidad de dicho método es el no reflejar el impacto de la congestión, pues los tiempos de viajes se tratan como estáticos. Centrado al método espacio-temporal, Kwan (1998) propone tres componentes: $i$ ) la referencia de actividad fija (cómo es medido 
el evento del individuo, desde y cuándo), ii) el conjunto de destinos y sus características (representado las oportunidades disponibles), y iii) el sistema de transporte que permita al individuo superar la separación espacio-temporal de las actividades. Sostiene que el emplear la red de transporte en los parámetros espacio-temporales, proporciona resultados más realistas para evaluar la accesibilidad respecto a las medidas geográficas clásicas.

\section{Metodología}

El proceso metodológico consta de cuatro pasos en dos enfoques para la estimación de los indicadores de accesibilidad. El primer enfoque, a partir del indicador de separación espacial, en términos generales considera la distancia que separa al origen del destino y un parámetro que representa la sensibilidad de la demanda ante los cambios en los costos de transporte. El segundo enfoque se basa en el indicador de interacción espacial, el cual incluye la dimensión de la oferta (como factor de atracción) y los costos de transporte (como factor de fricción). Para cada uno de estos enfoques se realizan dos escenarios, el primero considera el modo de transporte privado (automóvil), y el segundo el transporte público en autobús.

La investigación se centra en la obtención de los indicadores de accesibilidad, su comparación y evaluación, en consistencia con lo expuesto por Garrocho y Campos (2006), con la finalidad de contar con una metodología robusta que permita estimar indicadores de accesibilidad para definir políticas públicas en los procesos de planificación urbana en entornos periféricos. El nivel de agregación zonal básico es el área geoestadística básica (AGEB), área censal que sostiene Arbia (1989), y que es definida por el Instituto Nacional de Estadística y Geografía de México (INEGI, 2010) con los siguientes atributos: i) está delimitada por rasgos topográficos, ii) es homogénea en sus características económicas, sociales y geográficas, y iii) su extensión permite desplazamientos intrazonales a pie.

\subsection{Cálculo del Indicador de Separación Espacial}

Este tipo de indicador emplea la variable distancia entre el par origen-destino y la sensibilidad de la demanda ante los cambios en los costos de transporte (la fricción). En términos generales, estima el promedio de los recorridos entre todas las zonas de origen a todos los destinos posibles, empleando la Ecuación 1. 


$$
A_{i}=\frac{\sum_{j} c_{i j}}{d}
$$

donde:

$A_{i j}$ : indicador de accesibilidad (separación espacial)

$c_{i j}$ : costos de transporte entre pares $\mathrm{O}-\mathrm{D}$

$d$ : parámetro de fricción.

\subsubsection{Para el modo en vehículo privado (TPR)}

En términos generales es necesario obtener los costos de transporte y la variable de fricción de los costos, por ello se exponen a continuación los cuatro pasos para su obtención.

\section{Paso 1. Determinación de la distancia y el tiempo de viaje} en el ámbito periférico

Mediante la construcción de un grafo georreferenciado que representa la Zona Metropolitana de Querétaro (ZMQ) se obtuvo la distancia en cada arco (representación de cada vialidad). El tiempo de viaje para cada arco se obtuvo mediante la instrumentación de un sistema de posicionamiento global en un vehículo, con el cual se recorrieron las vialidades de la zona en periodos de flujo libre, registrando velocidades de punto cada cinco segundos y así poder obtener la velocidad media de recorrido para cada arco; dicho procedimiento está reportado en Obregón y Bueno (2015). En el Mapa 1 se muestran las isócronas de distancia y tiempo de viaje de la región periférica hacia la zona conurbada de Querétaro.

A partir de la información de distancia y tiempos de viaje, se aplica el algoritmo de Dijkstra (1959) para obtener la ruta más corta (en tiempo y distancia) entre todos los pares origen-destino. A partir de lo anterior se obtuvieron las matrices de distancia mínima $(M d m r)$ y de tiempos mínimos $(M z t)$.

\section{Paso 2. Estimación del valor del tiempo por motivo de trabajo (SHP)}

Para la obtención del valor del tiempo por motivo de trabajo se emplea la metodología propuesta por Torres y Hernández (2006) y actualizada en Torres et al. (2012), la cual permite estimar en términos económicos el valor del tiempo para los ocupantes de los vehículos que circulan por la red carretera de México, empleando la Ecuación 2. 


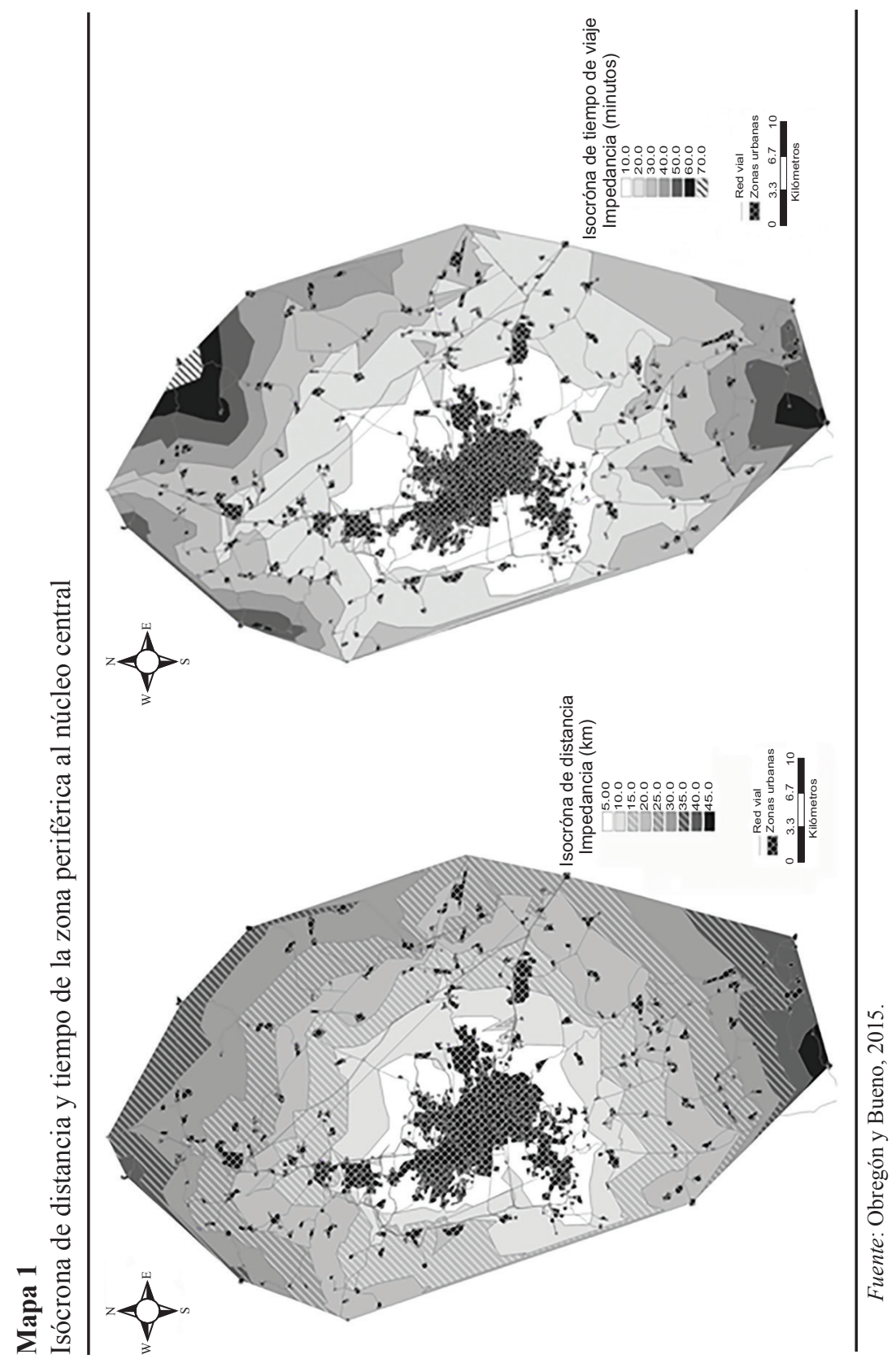




$$
S H P=\left[\frac{F I P^{*} S M G P * 7}{H T P}\right]
$$

donde:

SHP: valor del tiempo por motivo de trabajo

$H T P$ : promedio de las horas trabajadas por semana

$F I P$ : factor de ajuste del ingreso promedio de la población $S M G P$ : salario mínimo general promedio diario (en pesos).

Dichos parámetros son obtenidos considerando la región en la cual está inmerso nuestro ámbito de estudio (centro-occidente) de acuerdo con el Programa Nacional de Infraestructura 2007-2012 (SCT, 2007); se considera el SMGP para el año 2013 en 63.12 pesos (STPS, 2013). El FIP y el HTP regionalizado se obtienen a partir de Arroyo et al. (2010), siendo el FIP de 3.43 y el HTP de 41.08, con lo que resulta el SHP de 36.89 pesos / hora.

\section{Paso 3. Estimación del costo del transporte}

Para este análisis se consideraron los precios de las gasolinas Magna y Premium al valor vigente en el mes de enero de 2014, de 12.32 y $12.90 \mathrm{MXP} /$ litro, respectivamente. En cuanto al consumo de carburante en zonas metropolitanas, se contempló un rendimiento de $100 \mathrm{~km}$ por 11 litros de combustible, es decir de $9.09 \mathrm{~km}$ por litro. El rendimiento se ve afectado por las costumbres y hábitos de manejo, frenado y aceleración brusca. El costo del transporte se obtiene empleando la Ecuación 3. El resultado es una matriz cuadrada que nos indica el costo de transportarse entre cada par origendestino.

$$
c_{i j}=\left[\frac{M d m r / 1000}{R p c l} * C c\right]+\left[\left(\frac{M z t}{60}\right) * S H P\right]
$$

donde:

$c_{i j}:$ indicador de costos de transporte

Mdmr: matriz de distancia mínima

Rpcl: rendimiento promedio del carburante

$C c$ : costo del carburante

$M z t$ : matriz de tiempo mínimo

SHP: valor del tiempo por motivo de trabajo (en MXP por hora). 


\section{Paso 4. Parámetro de la fricción (impedancia)}

El parámetro de fricción $(d)$ para el indicador de accesibilidad se obtiene a partir de la estimación del modelo gravitacional, empleando la ecuación descrita en Ortúzar y Willumsen (2008: 270). Dicho parámetro representa un coeficiente de resistencia u oposición en la realización del viaje. A partir de lo anterior y mediante el empleo de la herramienta computacional TransCAD, se obtuvo la matriz que refleja la impedancia entre los pares origen-destino. Así, el modelo relaciona flujos entre zonas y la impedancia interzonal para realizar el viaje. El modelo gravitacional asume que el número de viajes producidos por la zona $i$ y atraídos por la zona $j$ es proporcional a: 1) el número de viajes producidos por la zona $i$;2) el número de viajes atraídos por la zona $j$, y 3) la función de impedancia entre zonas. Para su obtención se emplean las Ecuaciones 4 y 5 . Los datos de entrada de los viajes producidos y atraídos por medio de transporte en cada zona son obtenidos de la encuesta origen-destino reportada en Obregón et al. (2015 y 2016b).

$$
\begin{aligned}
& T_{i j}=P_{i} \frac{A_{j} f\left(d_{i j}\right)}{\sum_{\text {todaslaszonasz }} A_{z} f\left(d_{i z}\right)} \\
& T_{i j}=A_{j} \frac{P_{i} f\left(d_{i j}\right)}{\sum_{\text {todaslaszonasz }} P_{z} f\left(d_{j z}\right)}
\end{aligned}
$$

donde:

$T_{i j}$ : el flujo producido por la zona $i$ y atraído a la zona $j$

$P_{i}$ : el número de viajes producidos por la zona $i$

$A_{i}$ : el número de viajes atraídos por la zona $j$

$d_{i j}$ : la impedancia entre la zona $i$ y la zona $j$

$f\left(d_{i j}\right)$ : factor de fricción relacionado a la separación espacial entre la zona $i$ y la zona $j$.

Los valores derivados de la función de impedancia, denominados factores de fricción, se obtienen para cada par de zonas $i$ y $j$, y resultan de la matriz de factores de fricción. La función de impedancia empleada fue la exponencial (Ecuación 6) debido a que en su estimación y calibración se obtuvo una menor desviación y mejor convergencia; el proceso iterativo fue realizado empleando la herramienta computacional TransCAD. Los datos de entrada para obtener los factores de fricción parten de los costos de trans- 
porte estimados considerando lo expuesto en el Paso 3 para cada par origendestino. Un ejemplo numérico de su estimación empleando la Ecuación 6 se muestra en Ortúzar y Willumsen (2008: 276-278), y el proceso a detalle en William y McGuckin (1998: 36-47); sin embargo, dichos autores emplean en su ejemplo la función gamma (combinación de la función exponencial y potencial), y el procedimiento en el software empleado se detalla en Caliper (2015: 83-95).

$$
f(d)=e^{-c\left(d_{i j}\right)}
$$

donde:

$f\left(d_{i j}\right)$ : factor de fricción entre las zonas $i$ y $j$

$c$ : coeficiente del modelo

$d_{i j}$ : costo del viaje entre las zonas $i$ y $j$

$e$ : base de logaritmo natural.

\subsubsection{Para el modo en transporte público en autobús (TPU)}

Tal como se planteó para el caso del vehículo privado, el indicador emplea la Ecuación 1 en su estimación, considerando para ello la matriz del tiempo total de viaje en transporte público (incluyendo los tiempos obtenidos en la encuesta Origen-Destino, y correspondientes al Paso 1) y el valor del tiempo por motivo de trabajo (correspondiente al Paso 2); para ambos pasos se sigue el procedimiento ya descrito. Por lo anterior, a continuación se describe el procedimiento para estimar el costo de transporte (Paso 3) y el parámetro de impedancia (Paso 4).

Paso 3. Estimación de los costos de transporte

A diferencia del enfoque relativo al vehículo privado, en donde se consideraba la ruta más corta en tiempo sobre la red vial entre un origen y un destino, en el de transporte público se considera la línea o las líneas de autobús que toma el usuario para llegar a su destino. Con ello se incorpora el gasto por la tarifa del viaje en autobús y el tiempo total del recorrido, ambos declarados por el individuo en la encuesta origen-destino. Se consideró el valor del tiempo de viaje ya calculado de $36.89 \mathrm{MXP} /$ hora. A partir de los parámetros mencionados se obtienen los costos de transporte para cada par origendestino empleando la Ecuación 7. 


$$
c_{i j}=C v+\left[\left(\frac{T v}{60}\right) * S H P\right]
$$

donde:

$c_{i j}$ : indicador del costo de transporte

$C v$ : costo del viaje en transporte público

$T v$ : tiempo de viaje (en minutos)

SHP: valor del tiempo por motivo de trabajo (en MXP por hora).

Paso 4. Parámetro de ficción (impedancia)

Similar al procedimiento del vehículo privado, el parámetro de fricción $(d)$ se obtiene a partir de la estimación del modelo gravitacional, considerando los viajes en transporte público y empleando las Ecuaciones 4 y 5 . Tal como en el caso anterior, la función de impedancia empleada fue la exponencial (Ecuación 6) debido a que en su estimación y calibración se obtuvo una menor desviación y mejor convergencia.

\subsection{Indicador de interacción espacial}

Este indicador incluye la dimensión de la oferta (como factor de atracción) y los costos de transporte (es susceptible a cualquier variación), de tal manera que se puede estimar empleando la Ecuación 8.

$$
A_{i}=\sum \frac{O_{j}}{c_{i j}^{d}}
$$

donde:
$A_{i}$ : indicador de accesibilidad
$O_{j}$ : atractividad de la unidad de servicio
$c_{i j}$ : costo del transporte entre el origen y el destino
$d$ : parámetro de fricción.

Este tipo de indicador refleja las características de la demanda y la oferta en la zona de estudio y del sistema de transporte, y la atractividad es determinada por las unidades de servicio. Para este indicador, las actividades económicas son un parámetro de importancia, al analizar cómo es y cómo 
se manifiestan respecto a la atractividad de la unidad de servicio, respecto de su ubicación y en relación con los costos y el sistema de transporte.

Para determinar la atractividad de la unidad de servicio $\left(O_{j}\right)$ se consideran las unidades económicas de cada AGEB de la ZMQ, obtenidas del Directorio Estadístico Nacional de Unidades Económicas (DENUE) publicado por INEGI (2014). Una actividad económica se distingue de las demás por su función de producción, y su clasificación es determinada por la observación de su principal actividad. El Sistema de Clasificación Industrial de América del Norte (INEGI, 2013) define la actividad como: "aquella que en una determinada unidad económica y en un periodo de un año, genere más ingresos o en consecuencia la que más personal ocupe". La unidad económica es definida como el lugar o entidad donde se realizan las actividades económicas. Respecto al costo del transporte $\left(c_{i j}\right)$ y el parámetro de fricción (d) en vehículo privado y en transporte público, se empleó el procedimiento definido con anterioridad para el caso del indicador de separación espacial.

\section{Resultados y discusión}

El estudio de los niveles de accesibilidad permite determinar cuáles son las relaciones que existen entre la oferta y la demanda en una metrópoli considerando los viajes, sus tiempos, costos y actividades económicas, entre otros factores, y su relación con la elección del modo de transporte. Es necesario mencionar que la encuesta Origen-Destino se aplicó en 2012, por lo que las áreas con localidades construidas después de dicho año no se consideraron en esta investigación.

\subsection{Indicador de separación espacial}

A continuación se exponen los resultados obtenidos con el indicador de separación espacial, el cual incorpora la separación entre el par origen-destino y la sensibilidad de la demanda ante los cambios en los costos de transporte. En términos generales, estima el promedio de los recorridos entre todas las zonas de origen a todos los destinos posibles. Los resultados del indicador se contrastan con las tasas medias de producción de viajes en automóvil y autobús, así como con el ingreso promedio por familia. 


\subsubsection{Indicador de separación espacial para el transporte privado}

Se comparó el resultado de la accesibilidad para el trasporte privado empleando el indicador de separación espacial y la tasa media de producción de viajes por persona en automóvil (Gráfica 1). No se observa tendencia entre la accesibilidad y la tasa de viajes, pues se distingue cierta concentración en el valor de accesibilidad de separación espacial con valores bajos y altos en la tasa de producción de viajes. Al analizar las localidades dependiendo de su carácter (urbano o rural), tampoco se observa un patrón determinante, lo que indica que el indicador de accesibilidad no tiene una relación directa con la tasa de viajes, por lo que se decidió considerar el ingreso promedio por familia en cada zona.

La Gráfica 2 relaciona el indicador de accesibilidad por separación espacial, la tasa media de viajes y el ingreso promedio familiar, reflejando cómo el ingreso familiar presenta relación directa con la tasa de producción de viajes, mientras el indicador de accesibilidad muestra valores casi uniformes. Lo anterior indica que el contar con un vehículo propio propicia mayor participación a la zona metropolitana a través de la accesibilidad en la región.

\subsubsection{Indicador de separación espacial para el transporte público}

Al igual que en el apartado anterior, se relacionó el indicador de separación espacial con la tasa media de producción de viajes en transporte público y el ingreso promedio familiar. La Gráfica 3 contrasta el resultado del indicador de accesibilidad con la tasa media de producción de viajes en autobús, reflejando que: $i$ ) se presenta una agrupación de datos con un nivel bajo de producción de viajes y un bajo nivel de accesibilidad, y ii) respecto a las zonas rurales y urbanas, a diferencia del análisis de viajes en auto, se observa que las localidades rurales muestran tasas más altas de viajes en este medio. Si bien algunas zonas rurales exhiben niveles bajos de accesibilidad, en ellas los individuos tienden a desplazarse a zonas que están servidas por el sistema de transporte público. En este sentido, la accesibilidad está ligada a las tasas de viajes, ya que a medida que aumenta el indicador de accesibilidad los viajes aumentan.

Algunas de las localidades urbanas muestran valores más altos de accesibilidad, pero no todas muestran un alto nivel de ingreso; a la vez se observan zonas con un nivel alto de ingreso y baja accesibilidad. Así el ingreso familiar no es un factor determinante para este enfoque en transporte público, y sí lo es el nivel de accesibilidad. Lo anterior se refleja en la Gráfica 4, ya que a medida que aumenta el valor del indicador de accesibilidad, la producción de viajes se incrementa, pero a su vez, el ingreso promedio familiar no muestra relación. 


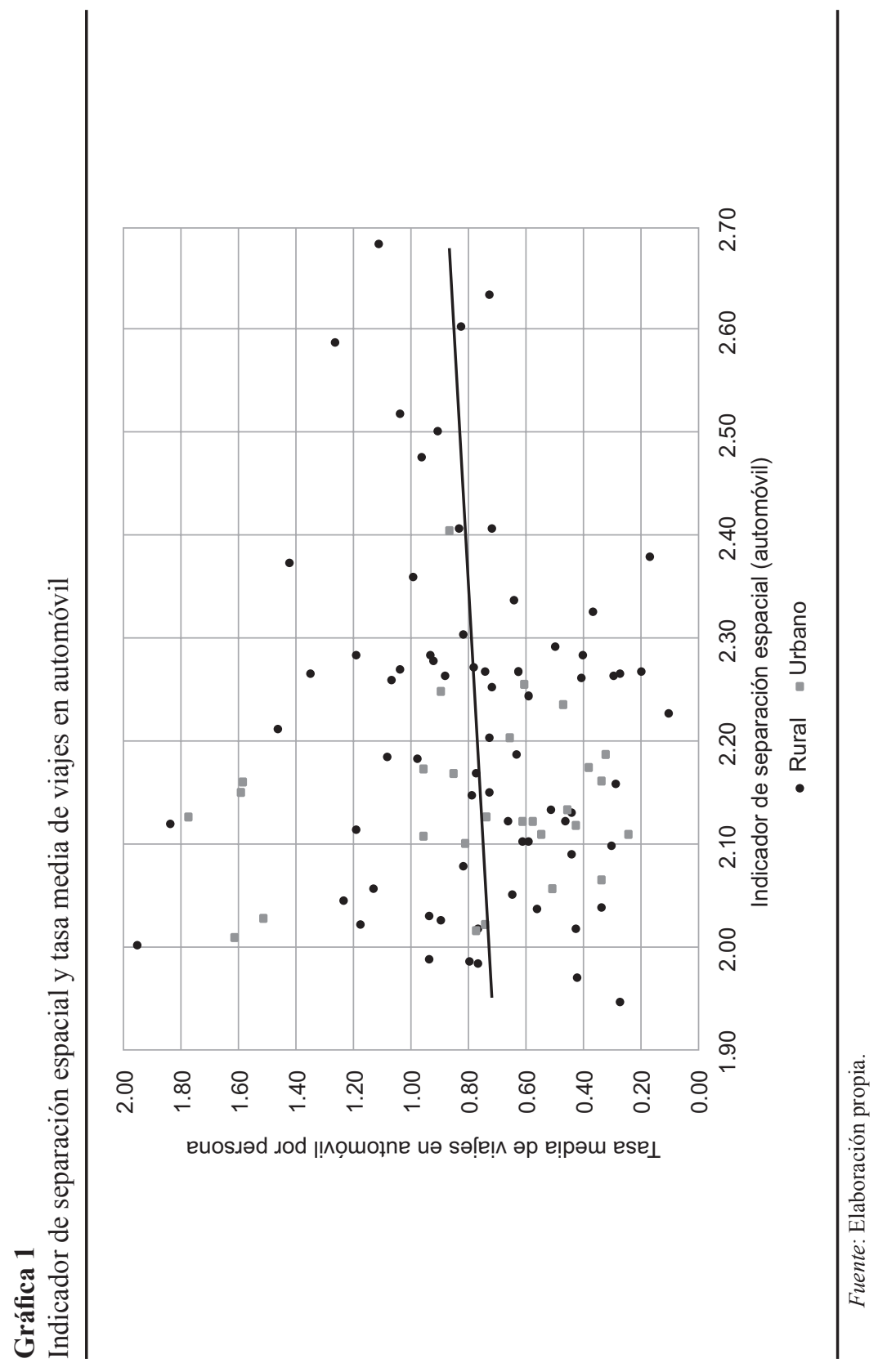




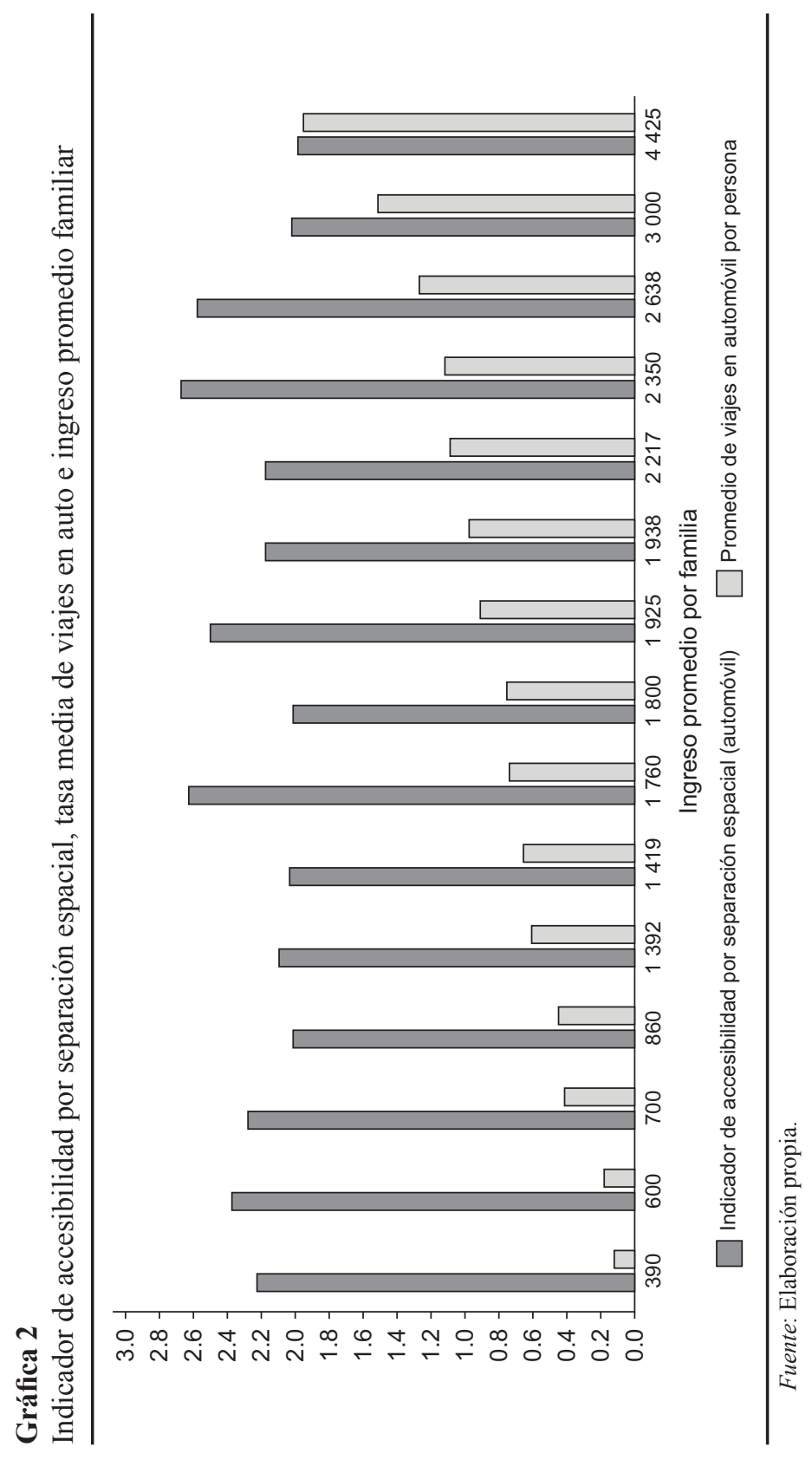




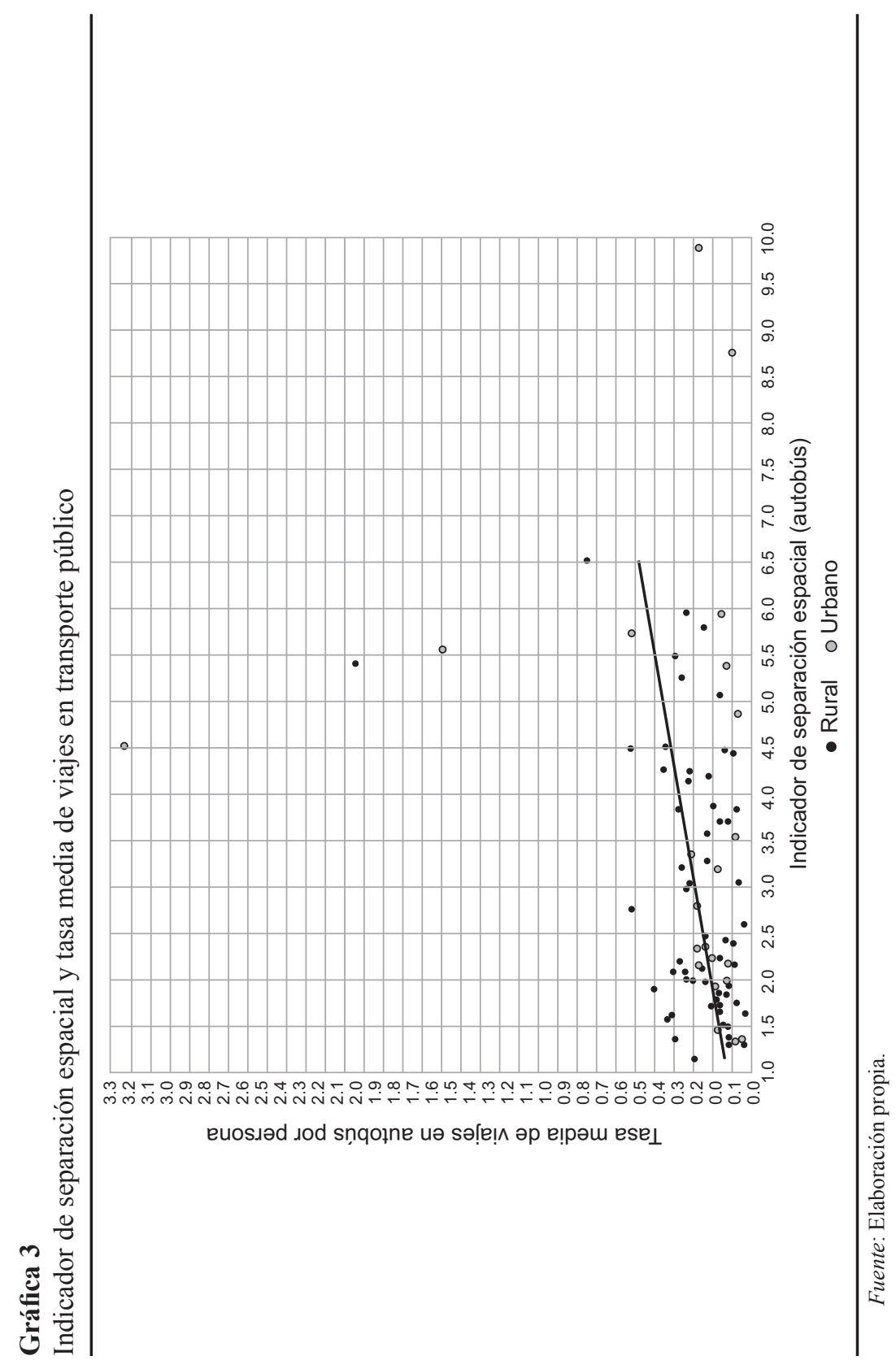




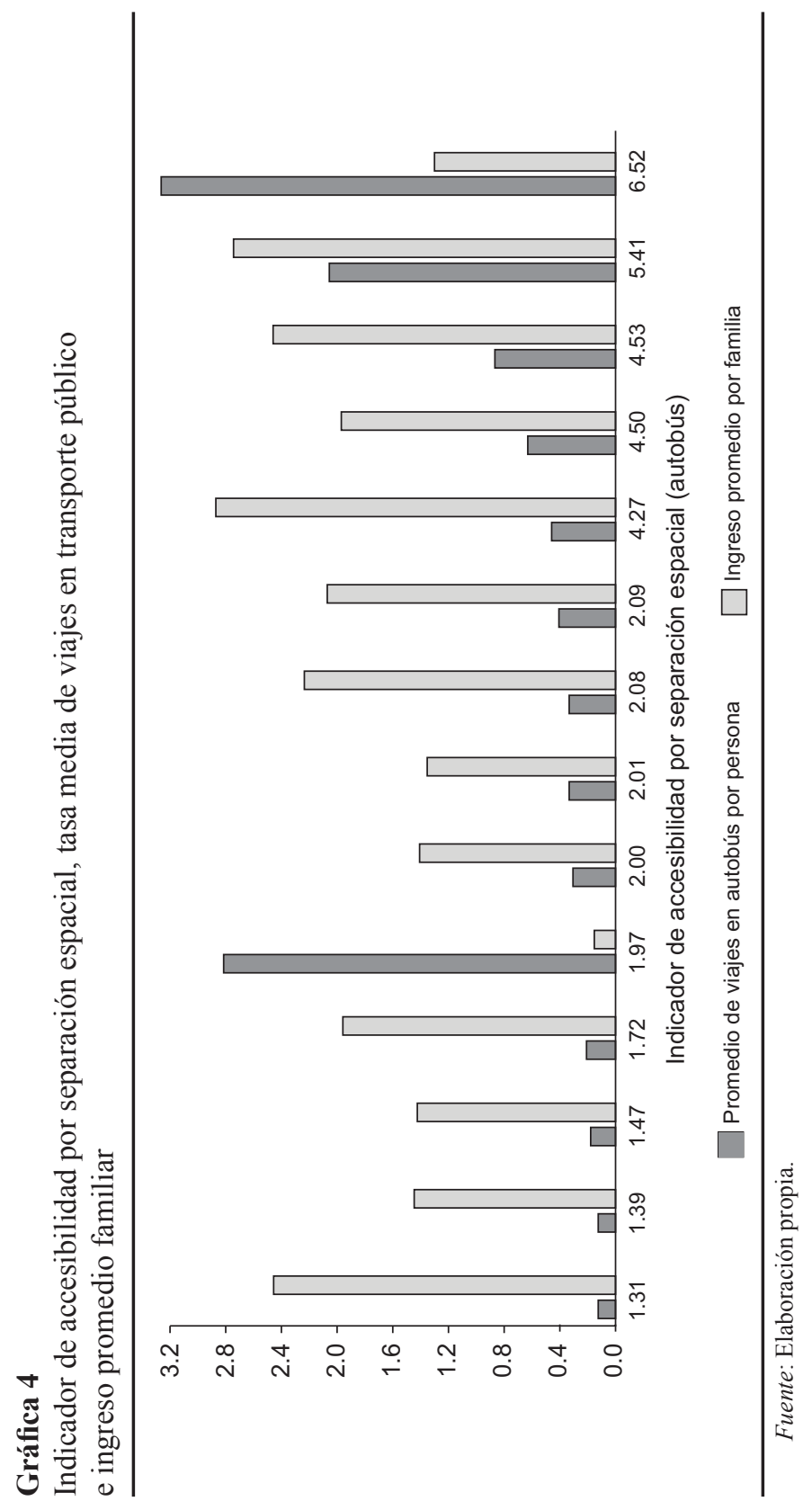




\subsection{Indicador de interacción espacial}

A continuación se exponen los resultados obtenidos con el indicador de interacción espacial, el cual incorpora las actividades económicas y la atractividad que ejercen las unidades de servicio de la zona conurbada en la periferia. Al igual que en los apartados anteriores, se considera el costo del viaje y la impedancia entre los pares origen-destino, contrastándose con las tasas medias de producción de viajes en automóvil y autobús, así como con el ingreso familiar promedio.

\subsubsection{Indicador de interacción espacial para el transporte privado}

La Gráfica 5 muestra el resultado del indicador de interacción espacial para el transporte privado respecto a la tasa media de producción de viajes, considerando las localidades rurales y urbanas. Se observa que las zonas con alta accesibilidad son las que presentan en su mayoría una tasa superior de generación de viajes en automóvil, siendo éstas principalmente de carácter urbano.

La mayor concentración de puntos se observa en los valores medios tanto para la accesibilidad como para la tasa promedio de viajes, por lo que en la Gráfica 6 se compara con el ingreso promedio por familia. Destaca una progresión ascendente en el valor del indicador con las tasas de viajes más altas; es decir, a medida que se incrementa el valor del ingreso y se tienen altos valores de accesibilidad, se reflejan mayores tasas de viajes.

\subsubsection{Indicador de interacción espacial para el transporte público}

La Gráfica 7 muestra los resultados de la estimación del indicador de interacción espacial para el transporte público, relacionados con la tasa media de viajes producidos por persona y el carácter (urbano o rural) de la localidad. Se observa que existe una concentración de puntos que no supera los 0.4 viajes por persona al día en transporte público, predominando menores tasas de generación de viajes en localidades urbanas, y siendo este tipo de localidades las que reflejan los mayores valores obtenidos en el indicador de interacción espacial.

De forma análoga a los análisis precedentes, en la Gráfica 8 se comparan las variables estimadas con el ingreso promedio por familia. En la Gráfica 8a se observa una relación directa del indicador de interacción espacial con el ingreso promedio por familia, es decir, las localidades periféricas con mayores ingresos son las que presentan a la vez valores más altos de accesibilidad. En la Gráfica 8b se relacionan las tres variables, observándose una 


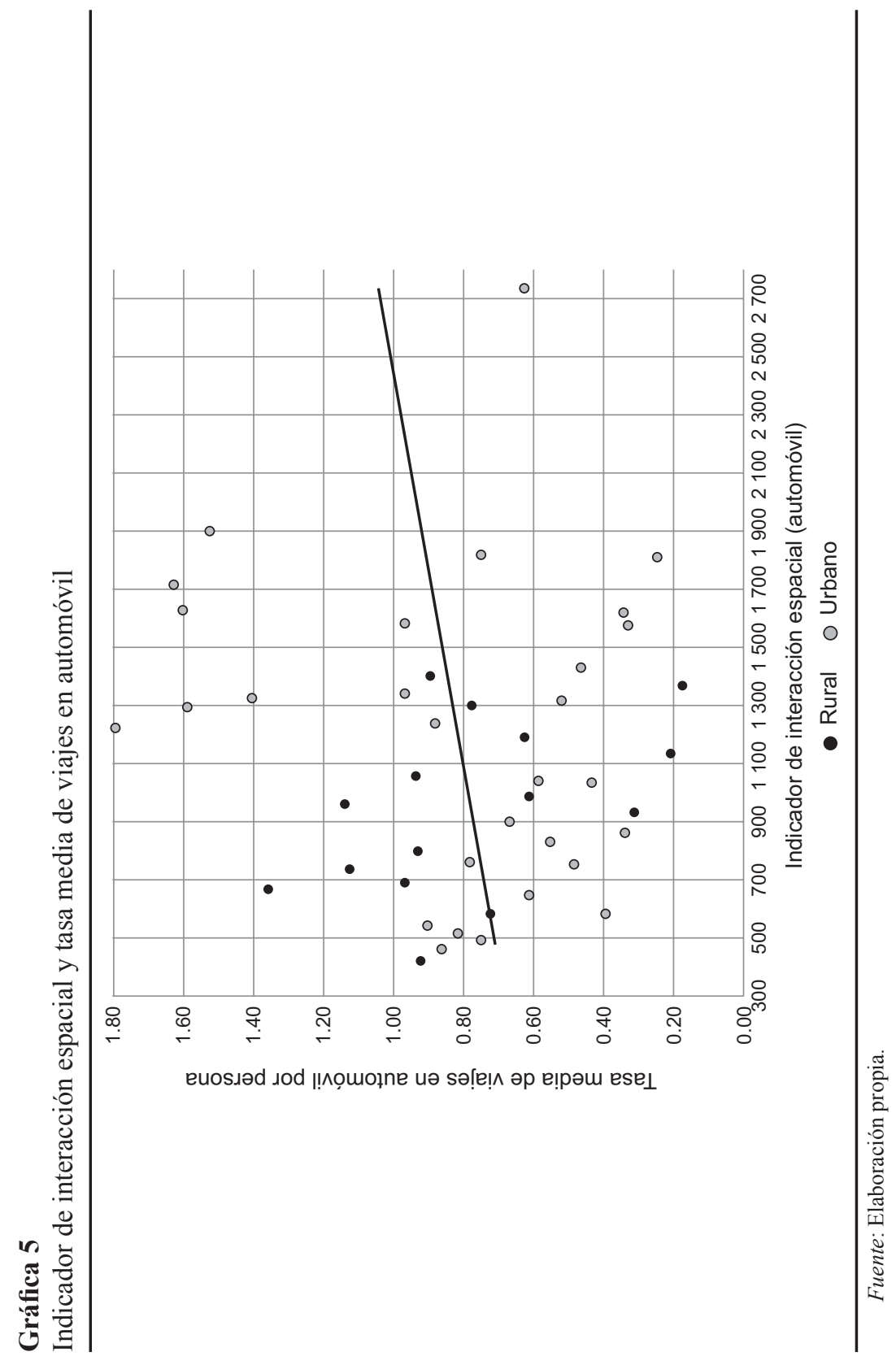




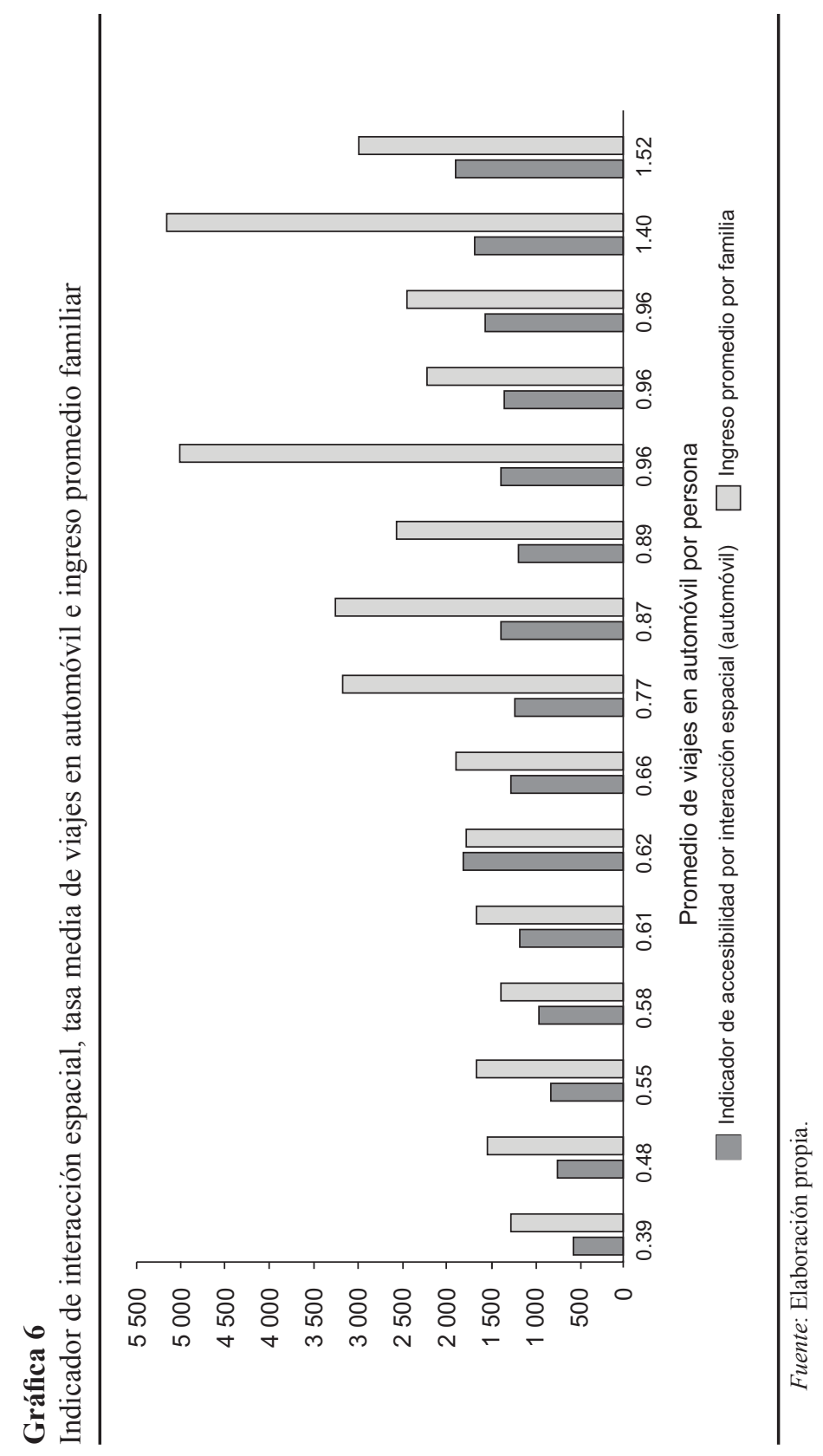




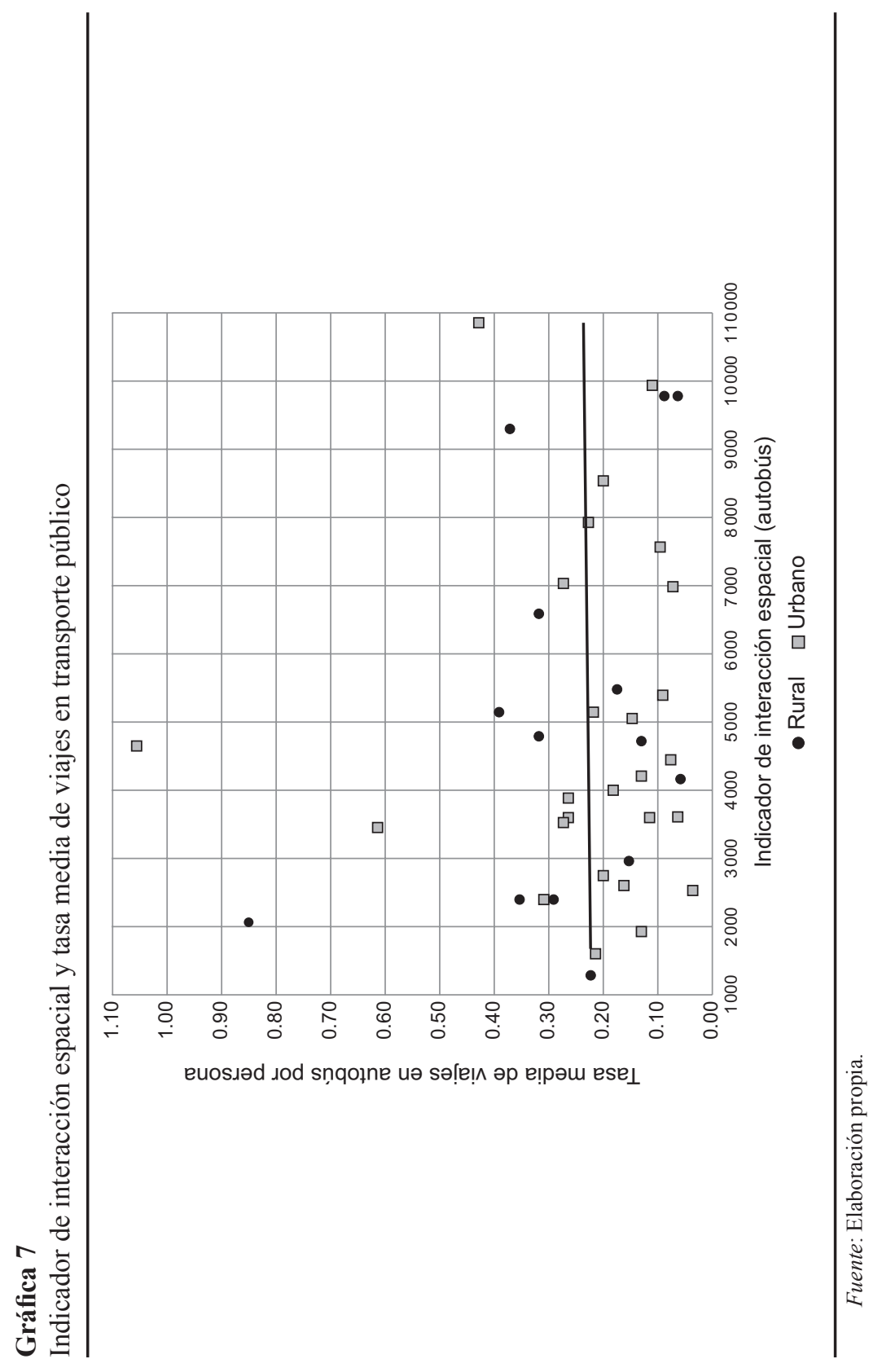




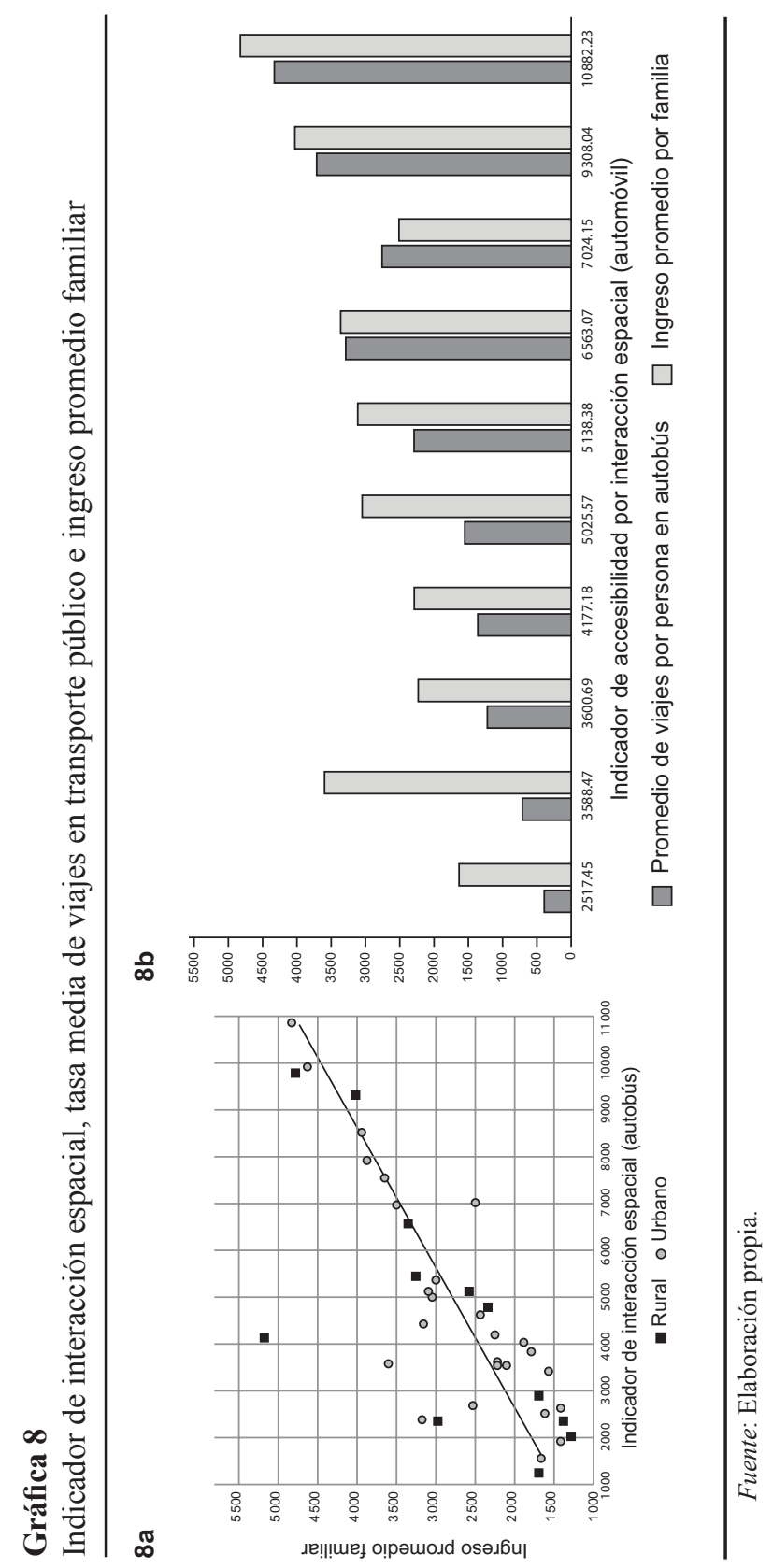


tendencia ascendente en el sentido de que, a medida que se incrementa el valor de accesibilidad, se presentan mayores tasas de generación de viajes y un mayor ingreso familiar.

\subsection{Comparación de indicadores y medios}

El Cuadro 1 muestra el resumen de los resultados obtenidos con cada indicador de accesibilidad considerando el automóvil y el autobús; se relacionan dichos indicadores y alternativas de medios con la tasa media de generación de viajes (TMGV) y el ingreso promedio por familia (IPF), para determinar qué características presentan una relación directa entre ellas y así comprobar la hipótesis.

En términos generales se observa que el indicador de interacción espacial refleja mayor coherencia entre el resultado obtenido y la tasa media de generación de viajes por persona. En el caso del indicador de separación espacial, el ingreso promedio por familia refleja mayor relación con la tasa de producción de viajes para el automóvil en comparación con el indicador. Mientras en el indicador de interacción espacial aplicado al caso del transporte público, las tres variables se muestran relacionadas. Por lo anterior, para el caso de estudio, de los dos indicadores analizados, el de interacción espacial es el que refleja con mayor fidelidad el comportamiento de la población y sus características económicas. Lo anterior deriva de que el indicador de interacción espacial incluye la dimensión de la oferta y los costos de transporte, mientras el de separación únicamente el costo. Por ello se recomienda a los planificadores el empleo de dicho indicador para definir las políticas públicas que potencialicen la accesibilidad en los entornos periféricos.

\section{Cuadro 1}

Resumen de resultados

\begin{tabular}{clccc}
\hline \multirow{2}{*}{$\begin{array}{c}\text { Indicador } \\
\text { de accesibilidad }\end{array}$} & \multirow{2}{*}{$\begin{array}{c}\text { Medio de } \\
\text { transporte }\end{array}$} & \multicolumn{3}{c}{ Variables relacionadas } \\
\cline { 3 - 5 } Separación espacial & Automóvil & Xccesibilidad & TMGV & IPF \\
& Autobús & $\checkmark$ & $\checkmark$ & $\checkmark$ \\
\multirow{2}{*}{ Interacción espacial } & Automóvil & $\checkmark$ & $\checkmark$ & X \\
& Autobús & $\checkmark$ & $\checkmark$ & X \\
& & & $\checkmark$ & $\checkmark$ \\
\hline
\end{tabular}

Fuente: Elaboración propia. 


\section{Conclusión}

Los resultados de la presente investigación confirman la hipótesis de partida, pues la accesibilidad en las áreas periurbanas de la Zona Metropolitana de Querétaro presenta relación directa con la tasa media de viajes en autobús. A partir de los enfoques para cada indicador de accesibilidad, tanto por separación espacial como para interacción espacial, se corrobora y se verifica la importancia de que la variable de accesibilidad está ligada a la elección del modo de transporte público en autobús.

En México, en las últimas décadas las administraciones están auxiliándose de encuestas de movilidad para definir sus políticas en dicha materia. Debido a su emergente proliferación, el presente procedimiento metodológico propuesto puede ser viable en la planeación de los entornos metropolitanos, considerando: i) los viajes con origen y destino entre zonas y medio de transporte; insumos necesarios para obtener ii) los tiempos de viaje entre zonas y por medio de transporte, el cual es un parámetro para la obtención del iii) costo de transportarse, que considera para el automóvil el valor del tiempo de la región y el consumo de carburante, mientras que para el transporte público, el tiempo de acceso, espera y a bordo de la unidad, así como la tarifa sufragada para realizar el viaje, y el caso de transbordo; con ello la estimación del iv) parámetro de la fricción (impedancia), que representa el coeficiente de resistencia u oposición en la realización del viaje, la cual aporta la variable espacial, mientras que la atractividad de las zonas para el indicador de interacción espacial considera v) las unidades de servicio en cada zona; así, se reflejan las características de la demanda, de la oferta y del sistema de transporte. El procedimiento planteado y su contraste con las tasas de viajes promedio por zona y medio de transporte permiten su interrelación y aplicación en entornos metropolitanos mexicanos.

A partir de los resultados obtenidos con el procedimiento propuesto, se recomienda considerar encuestas origen-destino para estimar la interacción entre zonas a partir de la estimación de un modelo gravitacional, y como medida de separación el costo generalizado del viaje por medio de transporte; en este sentido Kwan (1998) sostiene el empleo de la red de transporte. Sin embargo, se destaca la necesidad de incluir las características de explotación de los sistemas de transporte público dentro del costo generalizado del viaje (costo de transportarse), pues la zona puede estar dotada de infraestructura, pero puede presentar una baja cobertura o frecuencia del servicio. El proceso expuesto reafirma lo mencionado por Arbia (1989), en el sentido de no excluir unidades (zonas), además no se recomienda considerar a la

Estudios Demográficos y Urbanos, vol. 33, núm. 1 (97), 2018, pp. 111-147 ISSN 0186-7210; e-ISSN 2448-6515; doi: http://dx.doi.org/10.24201/edu.v33i1.1740 
población como variable de atracción, tal como establecen Bruinsma y Rietveld (1998), sino a las unidades económicas.

Comparando los dos indicadores, los resultados reflejan mayor coherencia para los dos medios de transporte analizados al emplear el indicador de interacción espacial. Para cada localidad, ya sea de carácter urbano o rural, los resultados reflejan que, aunque el ingreso promedio por familia en las zonas sea bajo, la población busca acceder a la conurbación para realizar sus actividades. Por lo anterior, en dichas zonas es necesario potencializar su accesibilidad, lo que generará mayor participación y posiblemente un mejor ingreso. En tal sentido, esta investigación puede auxiliar a la definición de políticas públicas para la dotación de infraestructura o sistemas de transporte para localidades que presenten bajos valores de accesibilidad e ingreso, lo cual influirá en mitigar la marginación de dichas zonas.

A partir de los resultados obtenidos, se plantean dos líneas de investigación futura. La primera (tomando en cuenta las críticas expuestas por Garrocho y Campos, 2006), plantear el enfoque propuesto en los indicadores de utilidad por medio de transporte, en específico al transporte público en autobús, considerando en la estimación del indicador el costo generalizado del viaje (incluyendo la tarifa, la valoración económica de los tiempos de viaje, la espera y el transbordo) e incorporando las restricciones del servicio; es decir, integrar parámetros de cobertura y restricciones en el número de transbordos, considerando para su definición los parámetros socioeconómicos de la zona de origen con la finalidad de acotar las alternativas de destino. Con ello se pueden establecer escenarios que mejoren los parámetros de explotación del sistema y cobertura, con el objetivo de reflejar el efecto de incrementar el número de destinos disponibles. La segunda línea de investigación propuesta es desarrollar parámetros de exclusión social con base en los indicadores de accesibilidad, ingreso y tasas de viajes; si bien el minimizar la marginación (que influye en que los individuos o grupos participen en las actividades normales de su sociedad) es un tema multidimensional, dichos parámetros pueden influir en su mitigación, bajo el supuesto de que las personas o los grupos de población están excluidos de cierto nivel mínimo de participación en las actividades por su situación geográfica, relacionada con su accesibilidad. 


\section{Bibliografía}

Alonso, Fernando (2007), “Algo más que suprimir barreras: conceptos y argumentos para una accesibilidad universal", Trans, Revista de Traductología, vol. 2, núm. 11, pp. 15-30. Recuperado de http://www.trans.uma.es/pdf/Trans_11/T.1530.FernandoAlonso.pdf

Arbia, Giuseppe (1989), Spatial data configuration in statistical analysis of regional economic and related problems, Dordrecht, Kluwer Academic Publishers.

Arentze, Theo A., Aloys W. J. Borgers y Harry J.P. Timmermans (1994), "Multistopbased measurements of accessibility in a GTS environment", International Journal of Geographical Information Systems, vol. 8, núm. 4, pp. 343-356. Recuperado de http://www.tandfonline.com/doi/abs/10.1080/02693799408902 005 ? journalCode $=$ tgis 19

Arroyo, José, Roberto Aguerrebere y Guillermo Torres (2010), Costos de operación base de los vehículos representativos del transporte interurbano 2010, Sanfandila, Querétaro, Instituto Mexicano del Transporte (Publicación Técnica, 337).

Bach, Lüder (1981), "The problem of aggregation and distance for analyses of accessibility and access opportunity in location-allocation models", Environment and Planning A, vol. 13, pp. 955-978. Recuperado de http://journals.sagepub. com/doi/abs/10.1068/a130955

Baradaran, Siamak y Farideh Ramjerdi (2001), "Performance of accessibility measures in Europe", Journal of Transportation and Statistics, vol. 4, núm. 2-3, pp. 31-48. Recuperado de https://www.rita.dot.gov/bts/sites/rita.dot.gov.bts/files/ publications/journal_of_transportation_and_statistics/volume_04_number_23/ paper 03/index.html

Berechman, Joseph y Robert Paaswell (2001), "Accessibility improvements and local employment: An empirical analysis”, Journal of Transportation and Statistics, vol. 4, núm. 2-3, pp. 49-66. Recuperado de https://www.rita.dot.gov/bts/ sites/rita.dot.gov.bts/files/publications/journal of transportation and statistics/ volume 04 number 23/paper_04/index.html

Bhat, Chandra, Susan Handy, Kara Kockelman, Hani Mahmassani, Qinglin Chen y Lisa Weston (2000), "Development of an urban accessibility index: Literature review", proyecto de investigación, Austin, Texas Department of Transportation / University of Texas, Center for Transportation Research.

Bhatta, Basudeb (2010), Analysis of urban growth and sprawl from remote sensing data, Nueva York, Springer.

Blanco, Ismael, Sonia Fleury y Joan Subirats (2012), "Nuevas miradas sobre viejos problemas: Periferias urbanas y transformación social”, Gestión y Política Pública, volumen temático, pp. 3-40. Recuperado de http://www.scielo.org.mx/ $\mathrm{pdf} / \mathrm{gpp} / \mathrm{v} 21 \mathrm{nspe} / \mathrm{v} 21 \mathrm{nspea1.pdf}$

Bruekner, Jan K. y David A. Fansler (1983), "The economics of urban sprawl: Theory and evidence on the spatial sizes of cities", The Review of Economics and Sta- 
tistics, vol. 65, núm. 3, pp. 479-482. Recuperado de http://www.gonzalo.depeco. econo.unlp.edu.ar/EU1UTDT/brueckner-fansler1983.pdf

Bruinsma, Frank y Piet Rietveld (1998), "The accessibility of European cities: theoretical framework and comparison of approaches", Environment and Planning $A$, vol. 30, pp. 499-521. Recuperado de https://www.researchgate.net/publica tion/23538673_The_accessibility_of_European_cities_Theoretical_framework and_comparison_of_approaches

Caliper Corporation (2015), Travel demand modelling with TransCAD Version 7 , Newton Massachusetts, Caliper Corporation.

CEAPAT (1996), Concepto europeo de accesibilidad, Madrid, Instituto de Migraciones y Servicios Sociales, Ministerio de Trabajo y Asuntos Sociales / Centro Estatal de Autonomía Personal y Ayudas Técnicas.

Currie, Graham, Tony Richardson, Paul Smyth, Dianne Vella-Broderick, Julian Hine, Karen Lucas, Janet Stanley, Jenny Morris, Ray Kinnear y John Stanley (2009), "Investigating links between transport disadvantage, social exclusion and wellbeing in Melbourne. Preliminary results", Transport Policy, vol. 16, núm. 3, Special Issue "International Perspectives on Transport and Social Exclusion", pp. 90-96. Recuperado de http://www.sciencedirect.com/science/article/pii/ S0967070X09000092

Dijkstra, Edsger W. (1959), "A note on two problems in connexion with graphs", Numerische Mathematik, vol. 1, pp. 269-271. Recuperado de http://www-m3. ma.tum.de/foswiki/pub/MN0506/WebHome/dijkstra.pdf

Domínguez, Trinidad, José Antonio Fraiz y Ma. Elisa Alén (2011), “Turismo y accesibilidad. Una visión global sobre la situación de España”, Cuadernos de Turismo, vol. 28, pp. 23-45. Recuperado de http://www.siis.net/documentos/ ficha/209780.pdf

Friedmann, John (2011), "Becoming urban: Periurban dynamics in Vietnam and China”, Pacific Affairs, vol. 84, núm. 3, pp. 425-434. Recuperado de https:// www.jstor.org/stable/23056183?seq=1\#page_scan_tab_contents

Fuentes, César (2009), "La estructura espacial urbana y accesibilidad diferenciada a centros de empleo en Ciudad Juárez, Chihuahua", Región y Sociedad, vol. 21, núm. 44, pp. 117-144. Recuperado de http://www.scielo.org.mx/pdf/regsoc/ v21n44/v21n44a5.pdf

Garrocho Rangel, Carlos F. y Juan Campos Alanís (2006), "Un indicador de accesibilidad a unidades de servicio clave para ciudades mexicanas: fundamentos, diseño y aplicación”, Economía, Sociedad y Territorio, vol. 6, núm. 22, pp. 1-60. Recuperado de http://www.redalyc.org/pdf/111/11162204.pdf

Geurs, Karst T. y Jan R. Ritsema van Eck (2001), Accessibility measures: Review and applications, Bilthoven, National Institute of Public Health and the Environment (RIVM Report, 408505 006). Recuperado de http://www.rivm.nl/ bibliotheek/rapporten/408505006.pdf

Goodall, Brian (1987), Dictionary of human geography, Harmondsworth, Penguin. 
Gutiérrez, Javier y Juan García (2006), "Movilidad por motivo trabajo en la comunidad de Madrid”, Revista del Instituto de Estudios Económicos, núm. 1-2, pp. 223-256. Recuperado de https://previa.uclm.es/cr/caminos/publicaciones/ Cuaderno_Ing_Territorio/4jornadas/JavierGutierrezPuebla/7.pdf

Guy, Clifford $\bar{M}$. (1983), "The assessment of access to local shopping opportunities: A comparison of accessibility measures", Environment and Planning B, vol. 10, pp. 219-238. Recuperado de http://journals.sagepub.com/doi/abs/10.1068/b10 0219? id=b100219\&journalCode=epba

Hägerstrand, Torsten (1975), "Space-time and human conditions", en Anders Karlqvist, Lars Lundqvist y Folke Snickars (coords.), Dynamic allocation of urban space, Teakfield, Farnborough, Hants, Saxon House, pp. 3-12.

Haller, Andreas (2014), “The 'sowing of concrete': Peri-urban smallholder perceptions of rural-urban land change in the central Peruvian Andes", Land Use Policy, vol. 38, pp. 239-247. Recuperado de http://ac.els-cdn.com/S0264837713002469/1s2.0-S0264837713002469-main.pdf?_tid=8f650dc4-7229-11e7-bb7d-00000aa b0f26\&acdnat $=1501091029$ 861b9e0620e2a050f6e6daa0988d98ac

Hamidi, Shima y Reid Ewing (2014), "A longitudinal study of changes in urban sprawl between 2000 and 2010 in the United States", Landscape and Urban Planning, vol. 128, pp. 72-82. Recuperado de http://www.sciencedirect.com/ science/article/pii/S0169204614001133

Handy, Susan L. y Kelly Clifton (2001), "Evaluating neighborhood accessibility: possibilities and practicalities", Journal of Transportation and Statistics, vol. 4, núm. 2-3, pp. 67-78. Recuperado de http://www.des.ucdavis.edu/faculty/handy/ JTS_paper.pdf

Handy, Susan L. y Deborah A. Niemeier (1997), "Measuring accessibility: An exploration of issues and alternatives", Environment and Planning A, vol. 29, pp. 11751194. Recuperado de http://journals.sagepub.com/doi/abs/10.1068/a291175

Harris, Briton (2001), “Accessibility: Concepts and applications”, Journal of Transportation and Statistics, vol. 4, núm. 2-3, pp. 15-30. Recuperado de https://www.rita. dot.gov/bts/sites/rita.dot.gov.bts/files/publications/journal_of_transportation_and statistics/volume_04_number_23/paper_02/index.html

INEGI (Instituto Nacional de Estadística y Geografía) (2010), Compendio de criterios y especificaciones técnicas para la generación de datos e información de carácter fundamental, Aguascalientes, México, INEGI.

INEGI (Instituto Nacional de Estadística y Geografía) (2013), Sistema de Clasificación Industrial de América del Norte, México, INEGI. Recuperado de http:// www.inegi.org.mx/est/contenidos/proyectos/SCIAN/presentacion.aspx (17 de marzo de 2014).

INEGI (Instituto Nacional de Estadística y Geografía) (2014), Directorio estadístico nacional de unidades económicas, México, INEGI. Recuperado de http://www. beta.inegi.org.mx/app/mapa/denue/ (9 de enero de 2015).

Johnson, Corey M. (2009), "Cross-border regions and territorial restructuring in 
Central Europe room for more transboundary space", European Urban and Regional Studies, vol. 16, núm. 2, pp. 177-191. Recuperado de http://journals. sagepub.com/doi/abs/10.1177/0969776409102190

Joseph, Allun E. y David Philips (1984), Accessibility and utilisation: Geographical perspectives on health care delivery, Londres, Harper \& Row.

Kwan, Mei-Po (1998), "Space-time and integral measures of individual accessibility: A comparative analysis using a point-based framework", Geographical Analysis, vol. 30, núm. 3, pp. 191-216. Recuperado de http://onlinelibrary.wiley. com/doi/10.1111/j.1538-4632.1998.tb00396.x/epdf

Lenntorp, Bo (1976), "Paths in space-time environments: A time geographic study of movement possibilities of individuals", Lund Studies in Geography, serie B, núm. 44, Estocolmo, Gleerup, pp. 150. Recuperado de http://www.popline.org/ node $/ 458894$

McFadden, Daniel (1974), "Conditional logit analysis of qualitative choice behavior", en Paul Zarembka (coord.), Frontiers in econometrics, Nueva York, Academic Press, pp. 105-142.

Miller, Harvey J. (1999), "Measuring space-time accessibility benefits within transportation networks: Basic theory and computational procedures", International Journal of Geographical Analysis, vol. 31, núm. 2, pp. 187-212. Recuperado de http://onlinelibrary.wiley.com/doi/10.1111/j.1538-4632.1999.tb00976.x/pdf

Miller, Harvey J. y Yi-Hwa Wu (2000), “GIS software for measuring space-time accessibility in transportation planning and analysis", GeoInformatica, vol. 4, pp.141-59. Recuperado de https://link.springer.com/content/pdf/10.1023 \%2FA\%3A1009820006075.pdf

Morris, Jennifer M., P.L. Dumble y Ramsay M. Wigan (1979), “Accessibility indicators for transport planning”, Transportation Research A, vol. 13, pp. 91-109. Recuperado de http://projectwaalbrug.pbworks.com/f/Transp+Accessib+-+Mo rris, +Dumble+and+Wigan+(1979).pdf

Nutley, Stephen (2003), "Indicators of transport and accessibility problems in rural Australia", Journal of Transport Geography, vol. 11, núm. 1, pp. 55-71. Recuperado de https://www.deepdyve.com/lp/elsevier/indicators-of-transport-andaccessibility-problems-in-rural-australia-yAn8MXq8e7

Ortúzar, Juan de Dios y Luis Willumsen (2008), Modelos de transporte, Santander, Universidad de Cantabria.

Obregón-Biosca, Saúl Antonio y Eduardo Betanzo (2015), “Análisis de la movilidad urbana de una ciudad media mexicana. Caso de estudio: Santiago de Querétaro", Economía, Sociedad y Territorio, vol. 15, núm. 47, pp. 61-98. Recuperado de http://www.scielo.org.mx/pdf/est/v15n47/v15n47a4.pdf

Obregón-Biosca, Saúl Antonio y César Omar Bueno-Ortiz (2015), "Dispersión urbana e integración funcional al núcleo central. Caso de estudio: Zona Metropolitana de Querétaro, México", Gestión y Política Pública, vol. 24, núm. 2, pp. 491-531. Recuperado de http://www.redalyc.org/articulo.oa?id=13341044006 Obregón-Biosca, Saúl Antonio, José Romero y Eduardo Betanzo (2015), “La movi- 
lidad en una zona metropolitana mexicana. Caso de estudio: Querétaro, México", Revista Transporte y Territorio, vol. 12, pp. 167-197. Recuperado de http:// revistascientificas.filo.uba.ar/index.php/rtt/article/view/1228

Obregón-Biosca, Saúl Antonio, Jorge Arturo Sánchez Escobedo y María de Lourdes Somohano Martínez (2016a), "Planificación de rutas turísticas para autobús a través de indicadores de accesibilidad integral y de dotación de bienes materiales e inmateriales", Revista Transporte y Territorio, vol. 14, pp. 144-166. Recuperado de http://www.redalyc.org/articulo.oa?id=333046307009

Obregón-Biosca, Saúl Antonio, José Romero, Juan Mendoza y Eduardo Betanzo (2016b), "Impact of mobility induced by urban sprawl: Case study of the Querétaro Metropolitan Area", Journal of Urban Planning and Development, vol. 142, núm. 2, 05015005. Recuperado de http://ascelibrary.org/doi/abs/10. 1061/(ASCE)UP.1943-5444.0000295

Ozbay, Kaan, Dilruba Ozmen y Joseph Berechman (2006), "Modeling and analysis of the link between accessibility and employment growth", Journal of Transportation Engineering, vol. 132, núm. 5, pp. 385-393. Recuperado de http:// ascelibrary.org/doi/full/10.1061/\%28ASCE\%290733-947X\%282006\% 29132\%3A5\%28385\%29

Paaswell, Robert y Jeffrey Zupan (2007), "Transportation infrastructure investments: New York and its global peers", Documento de Trabajo, Nueva York, City College of New York.

Pardo, Carlos (2005), "Salida de emergencia: Reflexiones sociales sobre las políticas del transporte", Universitas Psychologica, vol. 4, núm. 3, pp. 271-284. Recuperado de http://www.redalyc.org/html/647/64740302/

SCT (Secretaría de Comunicaciones y Transportes) (2007), Programa Nacional de Infraestructura 2007-2012, México, SCT.

STPS (Secretaría del Trabajo y Previsión Social) (2013), Salarios mínimos 2013 por área geográfica, México, Secretaría del Trabajo y Previsión Social / Comisión Nacional de los Salarios Mínimos (Conasami). Recuperado de http://www. conasami.gob.mx/nvos_sal_2013.html (20 de abril de 2014).

Thakuriah, Piyushimita (2001), "Introduction to the special issue on Methodological Issues in Accessibility Measures with Possible Policy Implications", Journal of Transportation and Statistics, vol. 4, núm. 2-3. Recuperado de https://www.bts. gov/archive/publications/journal_of_transportation_and_statistics/volume_04 number 23/introduction

Torres, Guillermo y Salvador Hernández (2006), Propuesta metodológica para la estimación del valor del tiempo de los usuarios de la infraestructura carretera en México: El caso del transporte de pasajeros, Querétaro, Instituto Mexicano del Transporte (Publicación Técnica, 291).

Torres, Guillermo, Salvador Hernández y Juan Ruvalcaba (2012), Actualización de la metodología para estimar el valor del tiempo de los usuarios de la red carretera nacional, Querétaro, Instituto Mexicano del Transporte (Publicación Técnica, 381).

Estudios Demográficos y Urbanos, vol. 33, núm. 1 (97), 2018, pp. 111-147 ISSN 0186-7210; e-ISSN 2448-6515; doi: http://dx.doi.org/10.24201/edu.v33i1.1740 
Valero, Ángeles (1984), "Movilidad espacial en Madrid", Anales de Geografía de la Universidad Complutense, vol. 4, Madrid, Universidad Complutense, pp. 207225. Recuperado de https://revistas.ucm.es/index.php/AGUC/article/viewFile/ AGUC8484110207A/32120

Weibull, Jörgen W. (1976), "An axiomatic approach to the measurement of accessibility”, Regional Science and Urban Economics, vol. 6, pp. 357-379. Recuperado de http://www.sciencedirect.com/science/article/pii/0166046276900314

William, Martin y Nancy McGuckin (1998), Travel estimation techniques for urban planning, Washington, D.C., Transportation Research Board, National Academy Press, pp. 170 (NCHRP Report, 365). Recuperado de https://ntl.bts.gov/ lib/21000/21500/21563/PB99126724.pdf

Zasada, Ingo, Christian Fertner, Annette Piorr y Thomas Nielsen (2011), "Peri-urbanisation and multifunctional adaptation of agriculture around Copenhagen", Geografisk Tidsskrift, vol. 111, núm. 1, pp. 59-72. Recuperado de http://orbit. dtu.dk/files/5797312/DJG_1-2011_WEB_59-72.pdf

Zhang, Bo (2004), Study on urban growth management in China, Beijing, Xinhua Press.

\section{Acerca de los autores}

Saúl Antonio Obregón-Biosca es ingeniero civil por la Universidad Autónoma de Querétaro (UAQ). Posee suficiencia investigadora en Urbanística, maestría en Ingeniería y doctorado en Ingeniería por el Departamento de Infraestructura del Transporte y Ordenación del Territorio de la Universidad Politécnica de Cataluña. Actualmente es profesor investigador en la División de Estudios de Posgrado de la Facultad de Ingeniería de la UAQ, en donde coordina la maestría en Vías Terrestres y Movilidad. Es miembro del Sistema Nacional de Investigadores, nivel II. Obtuvo el Premio Nacional "José Carreño Romaní" en 2016. Su línea de investigación actual se centra en el análisis de la movilidad metropolitana y las externalidades del transporte. Entre sus últimas publicaciones destacan: "Análisis de la movilidad urbana de una ciudad media mexicana, caso de estudio: Santiago de Querétaro" (en coautoría con Eduardo Betanzo-Quezada), Economía, Sociedad y Territorio, vol. 15, núm. 47, 2015; "Industrial location and national transport infrastructure: An empirical evidence between Queretaro and Tijuana metropolitan zones, Mexico" (en coautoría con Gerardo Ríos-Quezada), en Albert Tavidze (ed.), Progress in Economics Research, vol. 33, Nueva York, Nova Science, 2015; "Dispersión urbana e integración funcional al núcleo central. Caso de estudio: Zona Metropolitana de Querétaro, México" (en coautoría con César Omar Bueno-Ortiz), Gestión y Política Pública, vol. 24, núm. 2, 
2015; "Impact of mobility induced by urban sprawl: Case study of the Querétaro Metropolitan Area" (en coautoría con José A. Romero-Navarrete, Juan F. Mendoza-Sánchez y Eduardo Betanzo-Quezada), Journal of Urban Planning and Development, vol. 142, núm. 2, 2016. doi: 10.1061/(ASCE)UP.19435444.0000295 .

Marco Antonio Ángeles Espinosa es ingeniero civil por la Universidad Autónoma de Querétaro (UAQ). Desde el año 2013 colabora como investigador en el Laboratorio de Estudios Viales y Movilidad de la Facultad de Ingeniería de la UAQ.

Fecha de recepción: 23 de marzo de 2017.

Fecha de aceptación: 4 de julio de 2017. 
